\title{
Single mean arterial blood pressure drops during stroke thrombectomy under general anaesthesia are associated with poor outcome
}

\author{
Simon Fandler-Höfler ${ }^{1} \cdot$ Stefan Heschl ${ }^{2} \cdot$ Placido Argüelles-Delgado $^{2} \cdot$ Markus Kneihsl $^{1} \cdot$ Eva Hassler $^{3}$. \\ Marton Magyar ${ }^{3} \cdot$ Andreas Kainz $^{4} \cdot$ Andrea Berghold $^{4} \cdot$ Kurt Niederkorn $^{1} \cdot$ Hannes Deutschmann $^{3} \cdot$ Franz Fazekas $^{1}$. \\ Thomas Gattringer ${ }^{1}$ (D)
}

Received: 27 October 2019 / Revised: 7 January 2020 / Accepted: 9 January 2020 / Published online: 18 January 2020

(c) The Author(s) 2020

\begin{abstract}
Background We examined the influence of periprocedural blood pressure (BP), especially critical BP drops, on 3-month functional outcome in stroke patients undergoing mechanical thrombectomy (MT) under general anaesthesia (GA).

Methods We screened all patients with anterior circulation large vessel occlusion receiving MT under GA at our centre from January 2011 to June 2016 and selected those who had continuous invasive periinterventional BP monitoring. Clinical and radiological data were prospectively collected as part of an ongoing cohort study, monitoring data were extracted from electronic anaesthesia records. We used uni- and multivariable regression to investigate the association of BP values with unfavourable outcome, defined as modified Rankin Scale scores 3-6 3 months post-stroke.

Results 115 patients were included in this study (mean age 65.3 \pm 13.0 years, 55.7\% male). Periinterventional systolic, diastolic, and mean arterial BP (MAP) values averaged across MT had no effect on outcome. However, single BP drops were related to unfavourable outcome, with absolute MAP drops showing the highest association compared to both systolic and relative BP drops (with reference to pre-interventional values). The BP value with the strongest association with unfavourable outcome was identified as an MAP ever $<60 \mathrm{mmHg}(p=0.01)$ with a pronounced effect in patients with poor collaterals. An MAP $<60 \mathrm{mmHg}$ remained independently associated with poor functional outcome in multivariable analysis $(p<0.01)$. Conclusions For patients undergoing MT under GA, single MAP drops $<60 \mathrm{mmHg}$ are independently related to unfavourable 3-month outcome. Therefore, every effort should be made to prevent periinterventional hypotensive episodes, especially below this threshold.
\end{abstract}

Keywords Stroke $\cdot$ Large vessel occlusion $\cdot$ Thrombectomy $\cdot$ Blood pressure $\cdot$ Anaesthesia $\cdot$ Neurocritical care

Simon Fandler-Höfler and Stefan Heschl contributed equally to this manuscript.

Electronic supplementary material The online version of this article (https://doi.org/10.1007/s00415-020-09701-x) contains supplementary material, which is available to authorized users.

Thomas Gattringer

thomas.gattringer@medunigraz.at

1 Department of Neurology, Medical University of Graz, Auenbruggerplatz 22, 8036 Graz, Austria

2 Department of Anaesthesiology and Intensive Care Medicine, Medical University of Graz, Graz, Austria

\section{Introduction}

Mechanical thrombectomy (MT) has become the recommended treatment for acute ischemic stroke caused by anterior circulation large vessel occlusion (LVO) [1]. While there is no debate regarding a higher chance for improved outcome with successful recanalization, it is not yet clear if

3 Division of Neuroradiology, Vascular and Interventional Radiology, Medical University of Graz, Graz, Austria

4 Institute for Medical Informatics, Statistics and Documentation, Medical University of Graz, Graz, Austria 
MT should be performed under general anaesthesia (GA), conscious sedation (CS) or local anaesthesia alone. Recent single-centre randomized controlled trials found no difference in outcome between these approaches [2-4], while larger observational studies suggested a predominantly worse outcome for thrombectomy patients under GA [5, 6].

One reason for this could be the greater challenge of blood pressure management during the induction of GA where post-induction hypotension is common due to cardiodepressive and vasodilatory side effects of most anaesthetic agents [7]. There is, however, conflicting evidence whether hypotension during MT is independently related to worse outcome [8-12]. Previous studies on the relation of blood pressure (BP) during MT and outcome also vary in their suggestions of BP target levels and whether absolute BP levels or relative changes in $\mathrm{BP}$ from pre-intervention levels are more critical $[8,10]$. The 2018 AHA/ASA stroke guidelines note that BP should be kept $\leq 180 / 105 \mathrm{mmHg}$ during MT and recommend correction of hypotension and hypovolemia but do not indicate what minimal BP should be maintained [13]. Anaesthesiologic guidelines recommend preservation of a systolic BP $>140 \mathrm{mmHg}$ during MT, but this is not based on hard data [14]. It is also unclear whether systolic, diastolic or mean arterial blood pressure (SAP/DAP/MAP) has the greatest impact on outcome in the context of MT. Additionally, the influence of single critical BP drops during MT has not been investigated in depth and no specific cut-off values have been discovered yet. A commonly recommended minimal threshold for adequate cerebral blood flow autoregulation in the absence of increased intracranial pressure is a mean arterial pressure (MAP) $\geq 60 \mathrm{mmHg}$ [15]. Moreover, BP drops are likely particularly deleterious in patients with poor collaterals, but this has not been specifically investigated in MT patients thus far [16].

In view of these uncertainties, we explored the impact of different BP values (absolute and relative BP changes, SAP, DAP, and MAP) and their course during MT on outcome. Specifically, we hypothesized that critical BP drops of $\mathrm{MAP} \leq 60 \mathrm{mmHg}$ during MT were related to worse 3-month functional outcome. For this purpose, we analysed consecutive anterior LVO stroke patients who underwent GA for MT and had continuous invasive BP monitoring.

\section{Methods}

\section{Study participants}

For this retrospective cohort study, we identified all patients $\geq 18$ years who had received MT because of anterior circulation LVO stroke (i.e. occlusion of the intracranial internal carotid artery and/or middle cerebral artery in the M1 or M2 segments) between January 2011 and June 2016.
In our centre, MT is routinely performed by interventional radiologists using stent retrievers under GA by neuroanaesthesiologists. Type and doses of anaesthetic and blood pressure modifying drugs were individually chosen by the treating neuroanaesthesiologist. As we aimed for a uniform and well-characterized study cohort, patients were excluded from this study if they were intubated prehospitally or in the referring hospital or if they were not intubated at all during thrombectomy. Also, patients were excluded if electronic anaesthesia records were incomplete (BP measurement gaps of $\geq 5 \mathrm{~min}$ ). Paper-only anaesthesia records were excluded, as we aimed to only include exact and complete hemodynamic documentations. A flowchart of patient selection can be found in Fig. 1.

\section{Data assessment}

Clinical and radiological data were prospectively collected as part of an ongoing cohort study of all patients undergoing thrombectomy at our centre. Pre- and postinterventional Alberta stroke program early CT scores (ASPECTS) as well as collateral scoring on initial CT angiography using the TAN scale [17] were performed by two experienced neuroradiologists blinded to clinical data. MT was defined as successful if postinterventional Thrombolysis in cerebral infarction (TICI) scales of $2 b$ or more were achieved. Periinterventional vital signs (heart rate; systolic, diastolic and mean arterial $\mathrm{BP}$ and end-expiratory $\mathrm{CO}_{2}$, measured every minute) and anaesthesia management were extracted from electronic anaesthesia records, encompassing the time period from patient arrival in until departure from the angio suite (example shown in Fig. 2). BP measurement was done using invasive BP monitoring via peripheral arterial lines in all cases.

The primary outcome variable was functional neurological outcome at 3 months according to the modified Rankin Scale (mRS) and dichotomized as good (scores of 0-2) or unfavourable (scores 3-6). Furthermore, ordinal mRS shift analysis was performed. mRS scores were rated by stroke physicians during a scheduled visit at the stroke outpatient department.

\section{Statistical analysis}

Statistical analysis was performed using IBM SPSS Statistics 22. Normally distributed continuous variables were compared by the unpaired Student's $t$ test, for other distributions the Mann-Whitney $U$ test was used. For categorical variables, we used the Chi-square test and univariable logistic regression. $p$ values of less than 0.05 were considered statistically significant. We calculated receiver operating characteristic (ROC) curves for analysis of sensitivity and specificity of BP values towards 
Fig. 1 Study flowchart
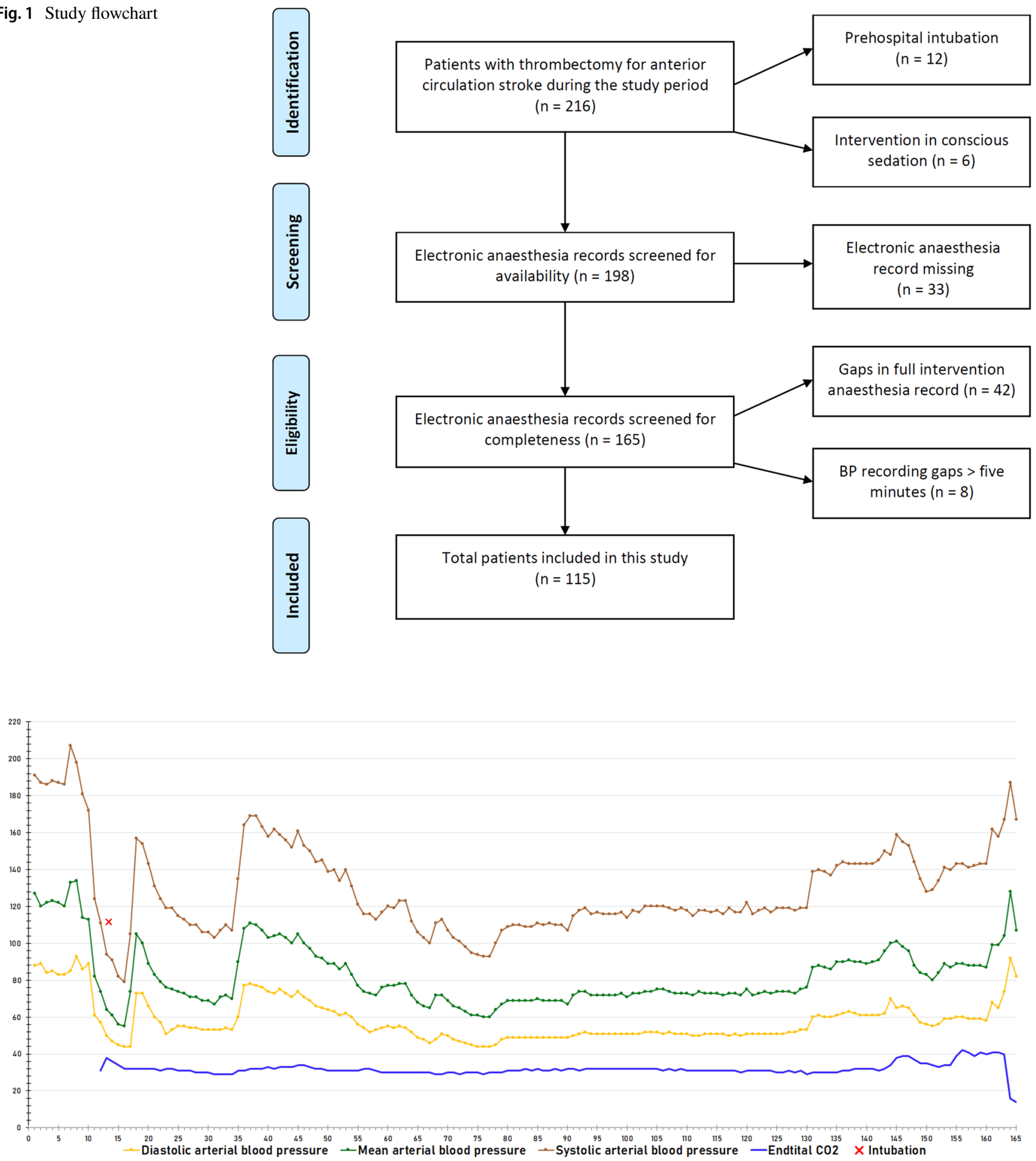

Fig. 2 Continuous electronic periinterventional blood pressure monitoring in a representative patient, showing a severe blood pressure drop after induction of anaesthesia (red cross)

functional outcome. All variables with $p<0.1$ in the univariable analysis were entered in a binary multivariable regression model.
The study was approved by the ethics committee of the Medical University of Graz. Anonymized datasets generated during this study are available from the corresponding author upon reasonable request. 


\section{Results}

\section{Patient characteristics and outcome}

From 216 patients who received MT for anterior LVO during the study period, 115 were included in this study. Patients had to be excluded because MT had been performed under conscious sedation $(n=6)$, intubation had been performed before arriving at our angio suite $(n=12)$, electronic anaesthesia records were not available $(n=33)$ or because of measurement recording gaps $(n=50$; Fig. 1$)$.

Their age ranged from 27 to 85 years (mean $65.3 \pm 13.0$ years) and $55.7 \%$ were men. There were no differences in clinical or radiological findings between included and excluded patients aside from slightly higher NIHSS scores in the latter (Table 1).
Most patients were treated for an occlusion of the M1-segment of the middle cerebral artery (75.7\%). Sixty percent received intravenous thrombolysis prior to MT. Median NIHSS at admission was 14, median pre-interventional ASPECTS was 9, successful recanalization (TICI $2 \mathrm{~b}-3$ ) was achieved in $83.5 \%$ of patients.

About half of the patients (51.3\%) had favourable 3-month functional outcome (mRS 0-2) and 14 patients $(12.2 \%)$ had died. Patients with unfavourable outcome (mRS 3-6) were older, had more severe stroke syndromes according to the NIHSS and more often had hypertension and diabetes. Worse collaterals on admission CT angiography and unsuccessful recanalization as well as lower pre- and especially postinterventional ASPECTS were also associated with poor 3-month outcome (Table 1).

Table 1 Clinical and radiological data of the study cohort regarding study inclusion and 3-month outcome

\begin{tabular}{|c|c|c|c|c|c|c|}
\hline & \multirow{3}{*}{$\begin{array}{l}\text { Study cohort } \\
n=115\end{array}$} & \multirow{3}{*}{$\begin{array}{l}\text { Excluded patients } \\
n=101\end{array}$} & \multirow[t]{3}{*}{$p$ value } & \multicolumn{3}{|l|}{ Study cohort } \\
\hline & & & & mRS 0-2 & mRS 3-6 & $p$ value \\
\hline & & & & $n=59(51.3 \%)$ & $n=56(48.7 \%)$ & \\
\hline \multicolumn{7}{|l|}{ Clinical data } \\
\hline Age $($ mean \pm SD $)$ & $65.3 \pm 13.0$ & $66.7 \pm 13.9$ & 0.43 & $62.1 \pm 13.8$ & $68.7 \pm 11.1$ & 0.001 \\
\hline Male sex & $64(55.7 \%)$ & $51(50.5 \%)$ & 0.53 & $31(52.5 \%)$ & $33(58.9 \%)$ & 0.49 \\
\hline Hypertension & $72(62.6 \%)$ & $67(66.3 \%)$ & 0.66 & $29(49.2 \%)$ & $43(76.8 \%)$ & 0.002 \\
\hline Dyslipidaemia & $17(14.8 \%)$ & $20(19.8 \%)$ & 0.33 & $7(11.9 \%)$ & $10(17.9 \%)$ & 0.37 \\
\hline Chronic heart disease $^{\mathrm{a}}$ & $17(14.8 \%)$ & $17(16.8 \%)$ & 0.55 & $5(8.5 \%)$ & $12(21.4 \%)$ & 0.05 \\
\hline Diabetes & $16(13.9 \%)$ & $23(22.8 \%)$ & 0.06 & $3(5.1 \%)$ & $13(23.2 \%)$ & 0.005 \\
\hline Atrial fibrillation & $48(41.7 \%)$ & $40(39.6 \%)$ & 0.85 & $22(37.3 \%)$ & $26(46.4 \%)$ & 0.32 \\
\hline Prestroke mRS (median, IQR) & $0(0)$ & $0(0)$ & 0.47 & $0(0)$ & $0(0)$ & 0.13 \\
\hline Stroke of unknown symptom onset & $27(23.5 \%)$ & $13(12.9 \%)$ & 0.05 & $11(18.6 \%)$ & $16(28.6 \%)$ & 0.21 \\
\hline NIHSS at admission (median, range) & $14(4-25)$ & $15(6-34)$ & 0.01 & $13(4-22)$ & $16(8-25)$ & $<0.001$ \\
\hline \multicolumn{7}{|l|}{ Radiological findings } \\
\hline MCA/M1-occlusion & $87(75.7 \%)$ & $76(75.2 \%)$ & 0.95 & $44(74.6 \%)$ & $43(76.8 \%)$ & 0.78 \\
\hline MCA/M2-occlusion & $13(11.3 \%)$ & $4(4.0 \%)$ & 0.05 & $7(11.9 \%)$ & $6(10.7 \%)$ & 0.85 \\
\hline Intracranial ICA occlusion & $11(9.6 \%)$ & $18(17.8 \%)$ & 0.08 & $5(8.5 \%)$ & $6(10.7 \%)$ & 0.68 \\
\hline CTA collateral scoring (TAN, median, range) & $2(0-3)$ & $2(0-3)$ & 0.16 & $2(0-3)$ & $1(0-3)$ & 0.04 \\
\hline ASPECTS pre-intervention (median, range) & $9(5-10)$ & $9(3-10)$ & 0.20 & $9(5-10)$ & $9(3-10)$ & 0.05 \\
\hline \multicolumn{7}{|l|}{ Intervention } \\
\hline Intravenous thrombolysis & $69(60 \%)$ & $68(67.3 \%)$ & 0.43 & $39(66.1 \%)$ & $30(53.6 \%)$ & 0.17 \\
\hline Time to groin puncture (min, median, IQR) & 200,82 & 205,84 & 0.56 & 203,81 & 197,74 & 0.55 \\
\hline Time to recanalization (min, median, IQR) & 260,93 & 271,90 & 0.36 & 254,92 & 272,77 & 0.68 \\
\hline Anaesthesia duration (min, median, IQR) & 112,64 & NA & NA & 108,54 & 131,67 & 0.02 \\
\hline Successful recanalization (TICI $2 b-3$ ) & $96(83.5 \%)$ & $82(81.2 \%)$ & 0.43 & $56(94.9 \%)$ & $40(71.4 \%)$ & 0.001 \\
\hline ASPECTS post-intervention $^{\mathrm{b}}$ (median, range) & $5(0-9)$ & $5(0-9)$ & 0.41 & $6(2-9)$ & $3(0-8)$ & $<0.001$ \\
\hline
\end{tabular}

ASPECTS Alberta stroke program early CT scores, CTA computed tomography angiography, ICA internal carotid artery, $M C A$ middle cerebral artery, NIHSS National Institutes of Health Stroke Severity Scale, $m R S$ modified Rankin Scale, TICI thrombolysis in cerebral infarction

${ }^{a}$ Coronary artery disease, heart failure, cardiomyopathy or valve disease

${ }^{\mathrm{b}}$ At follow-up imaging $24 \mathrm{~h}$ post-stroke 


\section{Periinterventional blood pressure}

Periinterventional BP drops were frequent. On average, the lowest periprocedural BP values recorded were 87, 46 and $60 \mathrm{mmHg}$ (SAP/DAP/MAP). When averaging BP values over the entire recording period, there was an average drop of about $40 \%$ compared to preinterventional values and single values were often much lower (Table 2).

In univariable analysis, preinterventional, average procedural and minimal BP values were not associated with 3 -month outcome. However, a periinterventional MAP that fell below $60 \mathrm{mmHg}$ at any time was associated with worse outcome (mRS 3-6 at 3 months post-stroke, $p=0.01$, OR $2.72,95 \%$ CI $1.26-5.85$, Table 2). Ordinal mRS shift analysis at 3 months also showed worse outcome for patients with an MAP drop $<60 \mathrm{mmHg}(p<0.01$, Fig. 3). There were no differences in demographic, clinical or radiological findings between patients whose periinterventional MAP dropped below $60 \mathrm{mmHg}$ or not (Table 3 ).

Using ROC curves to further investigate associations between BP values and functional neurological outcome, we found that absolute BP values were more relevant than relative values and that MAP showed a stronger association than SAP or DAP. In ROC curve analysis of absolute MAP drops, the strongest predictive value was confirmed as an MAP $<59.5 \mathrm{mmHg}$ at any time during MT (sensitivity 0.60 , specificity 0.63 , Youden's Index 0.23 , positive predictive value 0.60 , negative predictive value 0.63 , supplementary Fig. 1).
Table 2 Blood pressure values and frequency of drops in $\mathrm{MAP}<60 \mathrm{mmHg}$ related to 3-month outcome
Fig. 3 Modified Rankin Scale scores at 3 months of patients without and with periinterventional MAP drops below $60 \mathrm{mmHg}$, showing worse functional outcome of patients with such drops $(p<0.01)$

\begin{tabular}{|c|c|c|c|}
\hline & $\begin{array}{l}\text { mRS 0-2 } \\
n=59(51.3 \%)\end{array}$ & $\begin{array}{l}\operatorname{mRS} 3-6 \\
n=56(48.7 \%)\end{array}$ & $p$ value \\
\hline Preinterventional SAP $($ mean \pm SD) & $148.8 \pm 26.4$ & $151.9 \pm 26.5$ & 0.54 \\
\hline Preinterventional MAP $($ mean $\pm S D)$ & $105.9 \pm 19.7$ & $107.2 \pm 20.7$ & 0.73 \\
\hline Preinterventional DAP $($ mean \pm SD) & $80.4 \pm 14.0$ & $81.3 \pm 16.4$ & 0.75 \\
\hline Average SAP $($ mean \pm SD $)$ & $124.3 \pm 17.2$ & $122.6 \pm 17.4$ & 0.60 \\
\hline Average MAP $($ mean $\pm \mathrm{SD})$ & $82.2 \pm 12.1$ & $78.6 \pm 15.5$ & 0.17 \\
\hline Average DAP $($ mean $\pm \mathrm{SD})$ & $61.3 \pm 9.9$ & $58.8 \pm 8.5$ & 0.16 \\
\hline Minimal SAP $($ mean \pm SD) & $88.9 \pm 22.0$ & $85.6 \pm 18.6$ & 0.39 \\
\hline Minimal MAP $($ mean $\pm S D)$ & $61.7 \pm 12.2$ & $58.3 \pm 11.6$ & 0.13 \\
\hline Minimal DAP $($ mean $\pm S D)$ & $47.5 \pm 9.6$ & $44.8 \pm 8.3$ & 0.10 \\
\hline Lowest relative $\mathrm{SAP}^{\mathrm{a}}($ mean $\pm \mathrm{SD})$ & $58.2 \% \pm 16.3$ & $61.2 \% \pm 16.2$ & 0.32 \\
\hline Lowest relative $\mathrm{MAP}^{\mathrm{a}}($ mean $\pm \mathrm{SD})$ & $56.2 \% \pm 14.6$ & $60.0 \% \pm 15.0$ & 0.16 \\
\hline Lowest relative $\mathrm{DAP}^{\mathrm{a}}($ mean $\pm \mathrm{SD})$ & $57.2 \% \pm 14.8$ & $60.5 \% \pm 14.5$ & 0.22 \\
\hline Patients with MAP ever below $60 \mathrm{mmHg}$ & $27(45.8 \%)$ & $39(69.6 \%)$ & 0.01 \\
\hline
\end{tabular}

$D A P$ diastolic blood pressure, $M A P$ mean arterial pressure, SAP systolic blood pressure

${ }^{\text {a }}$ Relative to preinterventional values

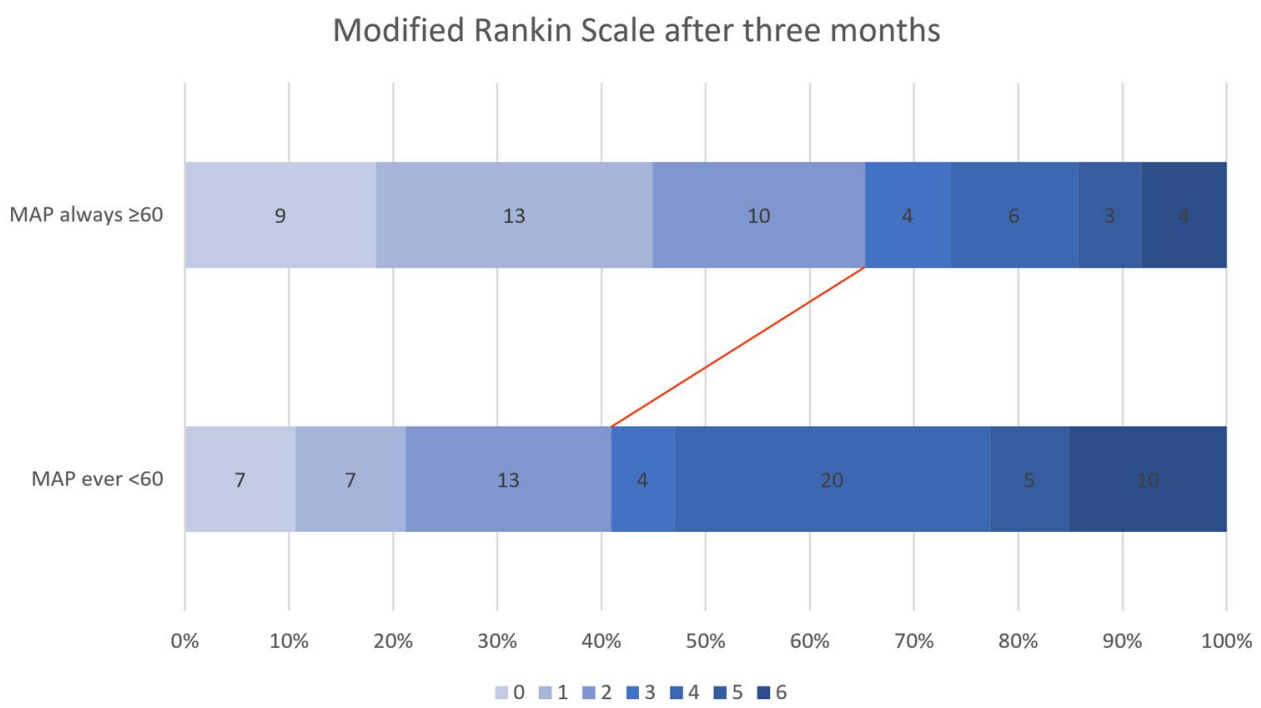


Table 3 Comparison of patients who had an MAP drop $<60 \mathrm{mmHg}$ during intervention or not

\begin{tabular}{|c|c|c|c|}
\hline & $\begin{array}{l}\text { MAP }<60 \mathrm{mmHg} \\
n=66(57.4 \%)\end{array}$ & $\begin{array}{l}\mathrm{MAP} \geq 60 \mathrm{mmHg} \\
n=49(42.6 \%)\end{array}$ & $p$ value \\
\hline \multicolumn{4}{|l|}{ Clinical data } \\
\hline Age $($ mean $\pm \mathrm{SD})$ & $66.3 \pm 12.6$ & $64.0 \pm 13.5$ & 0.36 \\
\hline Male sex & $37(56.1 \%)$ & $27(55.1 \%)$ & 0.92 \\
\hline Hypertension & $45(68.2 \%)$ & $27(55.1 \%)$ & 0.15 \\
\hline Dyslipidaemia & $13(19.7 \%)$ & $4(8.2 \%)$ & 0.09 \\
\hline Chronic heart disease ${ }^{b}$ & $8(12.1 \%)$ & $9(18.4 \%)$ & 0.35 \\
\hline Diabetes & $10(15.2 \%)$ & $6(12.2 \%)$ & 0.66 \\
\hline Atrial fibrillation & $26(39.4 \%)$ & $22(44.9 \%)$ & 0.55 \\
\hline Stroke of unknown symptom onset & $16(24.2 \%)$ & $11(22.4 \%)$ & 0.82 \\
\hline NIHSS at admission (median, range) & $14(4-25)$ & $15(5-23)$ & 0.82 \\
\hline \multicolumn{4}{|l|}{ Radiological findings } \\
\hline MCA/M1-occlusion & $49(74.2 \%)$ & $38(77.6 \%)$ & 0.68 \\
\hline MCA/M2-occlusion & $6(9.1 \%)$ & $7(14.3 \%)$ & 0.38 \\
\hline Intracranial ICA occlusion & $7(10.6 \%)$ & $4(8.2 \%)$ & 0.66 \\
\hline CTA collateral scoring (TAN, median, range) & $2(0-3)$ & $2(0-3)$ & 0.26 \\
\hline ASPECTS pre-intervention (median, range) & $9(5-10)$ & $9(3-10)$ & 0.45 \\
\hline \multicolumn{4}{|l|}{ Intervention } \\
\hline Intravenous thrombolysis & $36(54.5 \%)$ & $33(67.3 \%)$ & 0.17 \\
\hline Time to groin puncture (min, median, IQR) & 208,85 & 196,85 & 0.19 \\
\hline Time to recanalization (min, median, IQR) & 265,81 & 245,91 & 0.32 \\
\hline Anaesthesia duration (min, median, IQR) & 120,58 & 106,57 & 0.12 \\
\hline Successful recanalization (TICI 2b-3) & $54(81.8 \%)$ & $41(83.7 \%)$ & 0.86 \\
\hline \multicolumn{4}{|l|}{ Post-intervention } \\
\hline mRS 3 months post-stroke (median, range) & $4(0-6)$ & $2(0-6)$ & $<0.01$ \\
\hline Mortality at 3 months post-stroke & $10(15.2 \%)$ & $4(8.2 \%)$ & 0.26 \\
\hline \multicolumn{4}{|l|}{ Periinterventional blood pressure } \\
\hline Preinterventional SAP $($ mean \pm SD) & $150.4 \pm 28.7$ & $154.1 \pm 26.1$ & 0.49 \\
\hline Preinterventional MAP $($ mean \pm SD) & $105.5 \pm 21.9$ & $109.6 \pm 16.2$ & 0.30 \\
\hline Preinterventional DAP (mean $\pm \mathrm{SD}$ ) & $80.4 \pm 16.6$ & $81.4 \pm 13.2$ & 0.73 \\
\hline Minimal SAP (mean \pm SD) & $77.9 \pm 20.0$ & $100.0 \pm 12.5$ & $<0.001$ \\
\hline Minimal MAP $($ mean \pm SD) & $53.6 \pm 10.4$ & $68.9 \pm 7.6$ & $<0.001$ \\
\hline Minimal DAP (mean \pm SD) & $42.1 \pm 7.7$ & $51.6 \pm 7.8$ & $<0.001$ \\
\hline Average SAP $($ mean \pm SD) & $118.2 \pm 17.0$ & $130.6 \pm 15.0$ & $<0.001$ \\
\hline Average MAP $($ mean $\pm \mathrm{SD})$ & $76.3 \pm 10.8$ & $86.0 \pm 15.8$ & $<0.001$ \\
\hline Average DAP $($ mean $\pm \mathrm{SD})$ & $56.5 \pm 8.6$ & $65.0 \pm 7.9$ & $<0.001$ \\
\hline Lowest relative $\mathrm{SAP}^{\mathrm{a}}($ mean $\pm \mathrm{SD})$ & $54.0 \% \pm 16.4 \%$ & $67.7 \% \pm 12.5 \%$ & $<0.001$ \\
\hline Lowest relative $\mathrm{MAP}^{\mathrm{a}}($ mean $\pm \mathrm{SD})$ & $52.7 \% \pm 14.4 \%$ & $65.8 \% \pm 11.9 \%$ & $<0.001$ \\
\hline Lowest relative $\mathrm{DAP}^{\mathrm{a}}($ mean $\pm \mathrm{SD})$ & $54.2 \% \pm 13.3$ & $65.1 \% \pm 14.2$ & $<0.001$ \\
\hline
\end{tabular}

ASPECTS Alberta stroke program early CT scores, CTA computed tomography angiography, DAP diastolic blood pressure, ICA internal carotid artery, IQR interquartile range, $M A P$ mean arterial pressure, $M C A$ middle cerebral artery, NIHSS National Institutes of Health Stroke Severity Scale, $m R S$ modified Rankin Scale, SAP systolic arterial pressure, TICI thrombolysis in cerebral infarction

${ }^{a}$ Relative to preinterventional values

${ }^{\mathrm{b}}$ Coronary artery disease, heart failure, cardiomyopathy or valve disease
In patients with poor collaterals (TAN scale score $0-1,45.2 \%$ of patients), a periinterventional MAP below $60 \mathrm{mmHg}$ was even stronger associated with unfavourable 3 -month outcome (OR 4.29, 95\% CI 1.12-16.39, $p=0.03$ ) compared to patients with TAN scale scores $2-3$ (OR 1.88,
95\% CI 0.61-5.81, $p=0.28)$. Different lengths of hypotensive periods (both calculated as a continuous variable as well as dichotomized to $\geq 10 \mathrm{~min}$ compared to $<10 \mathrm{~min}$ ) were not related to clinical outcome. 


\section{Multivariable analysis}

In multivariable analysis comprising all variables with a $p$ value of $<0.1$ in univariable analysis, an MAP drop below $60 \mathrm{mmHg}$ remained an independent predictor for poor functional outcome at 3 months post-stroke (OR 6.17, 95\% CI $1.57-24.36, p<0.01)$, together with age, diabetes, chronic heart disease, NIHSS at admission, preinterventional ASPECTS and unsuccessful recanalization (detailed data in Table 4).

\section{Discussion}

In this retrospective cohort study, we found that MAP drops below $60 \mathrm{mmHg}$ during GA for MT were independently associated with worse functional outcome at 3 months poststroke. As ideal BP targets or thresholds for patients undergoing MT have not yet been agreed upon, our work adds several aspects to this debate.

The first important point is that our results suggest that absolute BP values have a stronger impact on neurological outcome than relative changes, both during induction of anaesthesia and throughout the whole thrombectomy procedure. Cerebral autoregulation helps to provide adequate blood flow to the brain across a range of BP values, but might be impaired in pathological conditions such as stroke $[18,19]$. Our results show the strongest association between unfavourable outcome and an MAP that dropped below $60 \mathrm{mmHg}$ at any point in time during MT. This could indicate the lower end of the autoregulatory capacity of the brain where cerebral blood flow becomes directly dependent on BP. It needs to be considered however, that the individual autoregulatory curve of patients might well be unpredictably shifted to the right, in particular in patients with long standing arterial hypertension and also differ between different cerebral pathologies [19].

The second important finding is that MAP drops showed a stronger association with worse outcome than systolic BP drops. Notably, MAP thresholds are not included in current recommendations for anaesthesiologic management of endovascular treatment for acute ischemic stroke [14]. From a pathophysiological point of view, MAP is the most important and commonly used blood pressure target value regarding cerebral blood flow [20]. Our clinical results now support this important role of mean over systolic BP. For clinical management of patients with low MAP, it needs to be considered, that diastolic pressure has a higher impact than systolic pressure. Therefore, elevation of diastolic pressure is probably the therapeutic approach of choice and could be achieved via fluid administration in many cases. However, this might be challenging in the neurointervention suite before the induction of anaesthesia for MT, in particular in patients with cardiac or renal comorbidities as it can lead to fluid overload and cardiac decompensation. Especially in clinically hypovolemic patients, early initiation of fluid administration already in the emergency department could potentially mitigate these risks. Disproportionate elevation of SAP by vasoconstrictor agents to increase MAP on the other hand, might potentially impede microcirculatory cerebral blood flow [21].
Table 4 Binary multivariable logistic regression analysis regarding poor outcome (mRS 3-6)

\begin{tabular}{|c|c|c|c|}
\hline Test variable & Odds ratio & $\begin{array}{l}95 \% \text { confidence } \\
\text { interval }\end{array}$ & $p$ value \\
\hline \multicolumn{4}{|l|}{ Clinical data } \\
\hline $\operatorname{Age}^{\mathrm{a}}$ & 1.07 & $1.01-1.13$ & 0.03 \\
\hline Hypertension & 1.02 & $0.25-4.26$ & 0.98 \\
\hline Diabetes & 16.3 & $1.17-225.9$ & 0.04 \\
\hline Chronic heart disease ${ }^{\mathrm{b}}$ & 9.29 & $1.31-65.96$ & 0.03 \\
\hline NIHSS at admission ${ }^{\mathrm{a}}$ & 1.27 & $1.07-1.49$ & $<0.01$ \\
\hline \multicolumn{4}{|l|}{ Radiological findings and intervention } \\
\hline ASPECTS pre-intervention ${ }^{\mathrm{a}}$ & 0.55 & $0.34-0.90$ & 0.02 \\
\hline CTA collateral scoring (TAN) ${ }^{\mathrm{a}}$ & 1.25 & $0.52-3.01$ & 0.61 \\
\hline Duration of anaesthesia & 0.99 & $0.98-1.01$ & 0.41 \\
\hline Unsuccessful recanalization (TICI 0-2a) & 23.2 & $2.17-247.9$ & $<0.01$ \\
\hline \multicolumn{4}{|l|}{ Periinterventional blood pressure drop } \\
\hline MAP ever below $60 \mathrm{mmHg}$ & 6.17 & $1.57-24.36$ & $<0.01$ \\
\hline
\end{tabular}

ASPECTS Alberta stroke program early CT scores, MAP mean arterial pressure, $m R S$ modified Rankin Scale, NIHSS National Institutes of Health Stroke Severity Scale

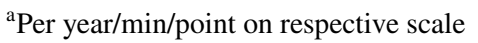

${ }^{\mathrm{b}}$ Coronary artery disease, heart failure, cardiomyopathy or valve disease 
The effect of a drop in MAP $<60 \mathrm{mmHg}$ regarding unfavourable outcome was quite strong in our study cohort andbesides well-established prognostic factors-remained significant in multivariable analysis. Furthermore, there were no baseline differences between patients who dropped to an $\mathrm{MAP}<60 \mathrm{mmHg}$ versus those who always had an MAP above this critical threshold. The strict avoidance of an MAP $<60 \mathrm{mmHg}$ should therefore be of paramount importance when GA is chosen for MT. This corresponds well with data from traumatic brain injury, showing that even a single episode of severe hypotension is associated with worse outcome [22]. While it could be assumed that longer duration of critical hypotensive periods leads to accumulated impact on patient outcome, we did not find such an effect. However, many hypotensive episodes in our study were short and the rather low number of longer hypotensive periods may have been too small to find statistically significant effects.

Notably, hypotensive episodes were not rare in our "real world" cohort; however, the severity and frequency of periinterventional hypotension was comparable to a previous retrospective study [8]. Post hoc analysis of the SIESTA trial in regards to $\mathrm{BP}$ values on the other hand revealed no association between periinterventional BP and functional outcome after 3 months [11]. However, this was a prospective trial investigating anaesthesia in MT and followed strict BP targets. Furthermore, single BP drops were not specifically explored. Therefore, the difference to our results may be explained by the different study designs and a more rigorous adherence to BP targets in the prospective study setting.

Our study may have unravelled a potential reason for the different performance of GA compared to CS for MT in small single-centre randomized controlled trials compared to observational and retrospective studies. Hypotension is more difficult to prevent in GA (especially during anaesthesia induction, after which most of the critical BP drops occurred in our study) and may occur more often (and more severe) in real-world patients compared to prospective studies which specifically investigated anaesthesia management of MT. This could be a possible—or at least partial—explanation why the prospective randomized controlled trials investigating anaesthesia for MT showed no difference in outcome between CS and GA [2-4], while observational and retrospective studies indicated worse outcomes under GA $[5,6]$.

Previous studies on anaesthesia management for MT have not particularly studied the pre-interventional collateralization status. Our finding that patients with worse collaterals are even more prone to worse outcome due to BP drops is in line with pathophysiological considerations [23].

This study has several strengths. All patients had invasive $\mathrm{BP}$ monitoring, which allowed us to precisely record BP. BP values were recorded and saved in the electronic anaesthesia records automatically. Patients with missing or incomplete records were excluded, the accuracy and data quality of BP measurements, the core variable for this study, can therefore be assumed to be very good. Notably, patients who were excluded from the study did not differ from study patients regarding important clinical baseline characteristics, except from higher NIHSS values in excluded patients, caused by the very high NIHSS values of prehospitally intubated patients (which were excluded from the study). Furthermore, clinical parameters were recorded prospectively in our study and we were able to include important radiologic parameters such as collateral status and pre- and postinterventional ASPECTS in our analysis.

Even though the retrospective nature of this study comes with known limitations, it also limits any possible observer effect in particular in regards to BP management, resulting in our study representing real-world data outside of wellcontrolled prospective trials on BP management in stroke. We did not explore the impact of different anaesthetic drugs and doses on both blood pressure and patient outcome as well as BP management before and after MT, which should be explored in further studies.

The uniformity of our study cohort limits the generalizability of our findings to other settings and particularly to other methods of anaesthesia (such as CS) for MT. Larger studies involving multiple centres and different anaesthetic management during MT are required to confirm our results and give further insights in periinterventional management of stroke patients.

Acknowledgements Open access funding provided by Medical University of Graz.

\section{Compliance with ethical standards}

Conflicts of interest The authors declare that they have no conflict of interest.

Ethical standards The study was approved by the ethics committee of the Medical University of Graz in accordance with the ethical standards laid down in the 1964 Declaration of Helsinki and its later amendments. Informed consent was waived by the ethics committee due to the retrospective nature of this study.

Open Access This article is licensed under a Creative Commons Attribution 4.0 International License, which permits use, sharing, adaptation, distribution and reproduction in any medium or format, as long as you give appropriate credit to the original author(s) and the source, provide a link to the Creative Commons licence, and indicate if changes were made. The images or other third party material in this article are included in the article's Creative Commons licence, unless indicated otherwise in a credit line to the material. If material is not included in the article's Creative Commons licence and your intended use is not permitted by statutory regulation or exceeds the permitted use, you will need to obtain permission directly from the copyright holder. To view a copy of this licence, visit http://creativecommons.org/licenses/by/4.0/. 


\section{References}

1. Goyal M, Menon BK, van Zwam WH et al (2016) Endovascular thrombectomy after large-vessel ischaemic stroke: a meta-analysis of individual patient data from five randomised trials. Lancet 387(10029):1723-1731

2. Simonsen CZ, Yoo AJ, Sørensen LH et al (2018) Effect of general anesthesia and conscious sedation during endovascular therapy on infarct growth and clinical outcomes in acute ischemic stroke: a randomized clinical trial. JAMA Neurol 75(4):470-477

3. Löwhagen Hendén P, Rentzos A, Karlsson J-E et al (2017) General anesthesia versus conscious sedation for endovascular treatment of acute ischemic stroke: the AnStroke Trial (Anesthesia During Stroke). Stroke 48(6):1601-1607

4. Schönenberger S, Uhlmann L, Hacke W et al (2016) Effect of conscious sedation vs general anesthesia on early neurological improvement among patients with ischemic stroke undergoing endovascular thrombectomy: a randomized clinical trial. JAMA 316(19):1986-1996

5. Brinjikji W, Pasternak J, Murad MH et al (2017) Anesthesiarelated outcomes for endovascular stroke revascularization: a systematic review and meta-analysis. Stroke 48(10):2784-2791

6. Campbell BCV, van Zwam WH, Goyal M et al (2018) Effect of general anaesthesia on functional outcome in patients with anterior circulation ischaemic stroke having endovascular thrombectomy versus standard care: a meta-analysis of individual patient data. Lancet Neurol 17(1):47-53

7. Südfeld S, Brechnitz S, Wagner JY et al (2017) Post-induction hypotension and early intraoperative hypotension associated with general anaesthesia. Br J Anaesth 119(1):57-64

8. Löwhagen Hendén P, Rentzos A, Karlsson J-E et al (2015) Hypotension during endovascular treatment of ischemic stroke is a risk factor for poor neurological outcome. Stroke 46(9):2678-2680

9. Takahashi CE, Brambrink AM, Aziz MF et al (2014) Association of intraprocedural blood pressure and end tidal carbon dioxide with outcome after acute stroke intervention. Neurocrit Care 20(2):202-208

10. Treurniet KM, Berkhemer OA, Immink RV et al (2018) A decrease in blood pressure is associated with unfavorable outcome in patients undergoing thrombectomy under general anesthesia. J Neurointerv Surg 10(2):107-111

11. Schönenberger S, Uhlmann L, Ungerer M et al (2018) Association of blood pressure with short- and long-term functional outcome after stroke thrombectomy: post hoc analysis of the SIESTA trial. Stroke 49(6):1451-1456
12. Pikija S, Trkulja V, Ramesmayer C et al (2018) Higher blood pressure during endovascular thrombectomy in anterior circulation stroke is associated with better outcomes. J Stroke 20(3):373-384

13. Powers WJ, Rabinstein AA, Ackerson T et al (2018) 2018 Guidelines for the early management of patients with acute ischemic stroke: a Guideline for Healthcare Professionals from the American Heart Association/American Stroke Association. Stroke 49(3):e46-e99

14. Talke PO, Sharma D, Heyer EJ, Bergese SD, Blackham KA, Stevens RD (2014) Republished: Society for Neuroscience in Anesthesiology and Critical Care expert consensus statement: Anesthetic management of endovascular treatment for acute ischemic stroke. Stroke 45(8):e138-150

15. Paulson OB, Waldemar G, Schmidt JF, Strandgaard S (1989) Cerebral circulation under normal and pathologic conditions. Am J Cardiol 63(6):2C-5C

16. Hindman BJ (2019) Anesthetic management of emergency endovascular thrombectomy for acute ischemic stroke, part 1: patient characteristics, determinants of effectiveness, and effect of blood pressure on outcome. Anesth Analg 128(4):695-705

17. Tan IYL, Demchuk AM, Hopyan J et al (2009) CT angiography clot burden score and collateral score: correlation with clinical and radiologic outcomes in acute middle cerebral artery infarct. Am J Neuroradiol 30(3):525-531

18. Petersen NH, Ortega-Gutierrez S, Reccius A, Masurkar A, Huang A, Marshall RS (2015) Dynamic cerebral autoregulation is transiently impaired for 1 week after large-vessel acute ischemic stroke. Cerebrovasc Dis 39(2):144-150

19. Rivera-Lara L, Zorrilla-Vaca A, Geocadin RG, Healy RJ, Ziai W, Mirski MA (2017) Cerebral autoregulation-oriented therapy at the bedside: a comprehensive review. Anesthesiology 126(6):1187-1199

20. Liu J, Zhu Y-S, Hill C et al (2013) Cerebral autoregulation of blood velocity and volumetric flow during steady-state changes in arterial pressure. Hypertension 62(5):973-979

21. Boerma EC, Ince C (2010) The role of vasoactive agents in the resuscitation of microvascular perfusion and tissue oxygenation in critically ill patients. Intensive Care Med 36(12):2004-2018

22. Chesnut RM, Marshall LF, Klauber MR et al (1993) The role of secondary brain injury in determining outcome from severe head injury. J Trauma 34(2):216-222

23. Shuaib A, Butcher K, Mohammad AA, Saqqur M, Liebeskind DS (2011) Collateral blood vessels in acute ischaemic stroke: a potential therapeutic target. Lancet Neurol 10(10):909-921 